\title{
Illness perceptions of gout patients and the use of allopurinol in primary care: baseline findings from a prospective cohort study
}

\author{
Ciaran P. Walsh, James A. Prior * (D, Priyanka Chandratre, John Belcher, Christian D. Mallen and Edward Roddy
}

\begin{abstract}
Background: Patients' perceptions of their illness are dynamic and can directly influence aspects of management. Our aim was to examine the illness perceptions of gout patients in UK primary care and associations with allopurinol use.

Methods: A health questionnaire was sent to 1805 people with gout aged $\geq 18$ years identified by a gout diagnosis or prescriptions for allopurinol or colchicine in their primary care medical records in the preceding 2 years. The questionnaire included selected items from the revised illness perception questionnaire (IPQ-R). Associations between illness perceptions and use of allopurinol were calculated using multinomial logistic regression adjusted for age, gender, deprivation status, body mass index, alcohol consumption, comorbidities and gout characteristics.

Results: One thousand one hundred eighty-four participants responded to the baseline questionnaire (65.6\%). Approximately half of responders perceived that they were able to control (51.2\%) or affect their gout through their own actions (44.8\%). Three quarters perceived treatments to be effective (76.4\%) and agreed that gout is a serious condition (76.4\%). Patients who agreed that they could control their gout (Relative Risk Ratio, $95 \%$ confidence interval 1.66 (1.12 to 2.45)) and that treatments were effective (2.24 (1.32 to 3.81)) were more likely to currently be using allopurinol than not using allopurinol. However, this significance was attenuated after adjustment for self-reported gout characteristics (1.39 (0.89 to 2.17 ) \& 1.78 (0.96 to 3.29) respectively).

Conclusions: Patients who perceive that they can control their gout and that treatments are effective are more likely to be using allopurinol, this suggests that better information is needed for the patient from GPs and rheumatologist to reassure and support their use of ULT.
\end{abstract}

Keywords: Allopurinol, Epidemiology, Gout, Illness perception, Primary care

\section{Background}

Gout is the most prevalent form of inflammatory arthritis, affecting $2.5 \%$ of adults [1]. Gout is primarily attributed to hyperuricemia, the saturation of urate in and around the joints and connective tissues. Deposition of mono-sodium urate (MSU) crystals then leads to attacks of acute gout, tophi and progressive joint damage [2].

Long-term treatment of gout is achieved through urate-lowering therapies (ULT), such as allopurinol, which is the recommended drug for initial lowering of serum urate (sUA). Once target sUA levels have been

\footnotetext{
* Correspondence: j.a.prior@keele.ac.uk

Research Institute for Primary Care \& Health Sciences, Keele University, Staffordshire ST5 5BG, UK

reached, allopurinol use should be continued throughout life, to maintain sUA levels [3]. Despite these clear guidelines, primary care management of gout is typically sub-optimal in primary care [1].

When individuals are diagnosed with a chronic condition, such as gout, they will develop organised patterns of beliefs in response. Depending on how the individual perceives this new illness, these can directly influence aspects of management, such as drug adherence, but such illness perceptions are also dynamic and can alter [4]. Illness perceptions can be measured through rating and assessment of questionnaire statements related to the impact of illness on the individual, such as the 'Revised Illness Perception Questionnaire' (IPQ-R) [5]. 
Responses measure how the individual components of illness perception, for example Identity (Is illness central to how the individual identifies themselves?), Cause (How the disease arose?), interact to form the patient's overall opinion regarding their chronic condition [6]. How one interprets the individual components forms an overall illness perception [7].

Illness perceptions are dynamic, with the potential to change depending on alterations in beliefs. Perceptions may change due to internal factors (e.g. beliefs about disease control) or external factors (e.g. effects of medication) and can potentially result in changes to disease outcomes [8]. De Gucht [9] found the relationship between irritable bowel symptom severity and separate quality of life dimensions to be fully mediated by illness perceptions. Dissatisfaction with treatment can manifest in follow-up consultations and lead to reduced adherence to treatment, leading to poorer disease outcomes [10]. Conversely an alteration in thoughts and emotions can also improve outcomes [4].

The relationship between illness perceptions and disease outcomes in gout has only previously been examined in a single study. Dalbeth et al. recruited 142 patients from primary and secondary care in New Zealand who completed the Brief Illness Perception Questionnaire (B-IPQ) and Health Assessment Questionnaire (HAQ II), at baseline and 1-year follow up. Participants who had poorer illness perceptions had an increased likelihood of disease progression and poorer adherence to ULT, suggesting that identification of such illness perceptions in clinical practice could be important to potentially identify those at risk of worse outcome [8]. However, the small sample size used in the Dalbeth et al. paper and the inclusion of secondary care patients, who would be likely to have more severe disease and hence different illness perceptions, are unlikely to be comparable to gout patients from UK primary care. $25 \%$ of participants had tophi, which is more than typically seen in UK primary care.

There is presently little data on the illness perceptions of gout patients. Therefore, the aim of this cross-sectional analysis was to examine the illness perceptions of gout patients from UK primary care, with the specific objective to examine the associations between illness perceptions and the current use of allopurinol.

\section{Methods}

\section{Study design and population}

In 2012, as part of a longitudinal cohort study, a baseline questionnaire was mailed to 1805 gout patients, aged 18 years of age and older, registered with 20 general practices. Gout was defined by a history of previous gout consultation or a prescription of allopurinol or colchicine within the 2 years, identified in primary care medical records, prior to completing the baseline questionnaire [11].
This paper will describe cross-sectional analysis of baseline data from the cohort study. This study was approved by the North West - Liverpool East Research Ethics Committee (Ref no: 12/NW/0297).

\section{Survey measures}

At baseline, characteristics recorded through the selfreport questionnaire included age, gender, and body mass index (BMI), calculated from self-reported height and weight. Indices of Multiple Deprivation (IMD) were used to quantify deprivation status and categorise individuals into tertiles of; $20 \%$ least affluent, $20 \%$ most affluent and middle $60 \%$. We also recorded participants' alcohol consumption.

Several gout specific characteristics were assessed, including; number of gout attacks in the previous 12 months, history of attacks of gout affecting more than one joint (oligo/polyarticular attacks), age at gout diagnosis, whether participants were currently experiencing a gout attack and whether they were currently taking allopurinol. Self-reported comorbidities were recorded, including; hypertension, transient ischaemic attack, anxiety, myocardial infarction, hyperlipidaemia, kidney stones and type II diabetes mellitus. The prevalence and severity of anxiety and depression were each recorded using a specific disease outcome, the generalised anxiety disorder (GAD-7) and patient health questionnaire (PHQ-9), respectively.

Questions were selected from the Revised Illness Perception Questionnaire (IPQ-R) [5], with participants answering several questions related to gout, comprising; i) there is a lot I can do to control my gout?, ii) what I do will affect my gout?, iii) treatments are effective?, iv) gout is serious?, in order to determine illness perceptions and patient understanding of disease.

\section{Statistical analysis}

The characteristics of the study sample were initially summarised using descriptive statistics. The mean age (standard deviation (SD)) and gender were reported. BMI was categorised by those with a score $<25 \mathrm{~kg} / \mathrm{m}^{2}$ (healthy weight) or $\geq 25 \mathrm{~kg} / \mathrm{m}^{2}$ (overweight). The frequency with which alcohol was consumed was categorised as; i) never, ii) occasionally, iii) 1-3 times per month, iv) 1-2 times a week, v) 3-4 times a week or vi) daily/almost daily. For gout-specific characteristics, number of gout attacks in the preceding 12 months was categorised into i) 0 gout attacks, ii) $1-3$ gout attacks, iii) $\geq 4$ gout attacks. History of oligo/polyarticular attacks and allopurinol use were categorised as yes or no. Possible IPQ-R responses were strongly agree, agree, uncertain, disagree or strongly disagree to the four statements. 'Strongly agree' and 'Agree' were combined into a single category ('Agree') and 'Strongly disagree' and 'Disagree' into a single category 
'Disagree'. Collapsing of categories was performed, boosting the number of responses in each of the three remaining categories and reducing extreme differences in response numbers between individual categories for each question.

Associations between the samples' characteristics and the use of allopurinol were initially compared using independent $t$-test or chi-squared test $\left(\chi^{2}\right)$. Multinomial logistic regression was used to identify associations between the use of allopurinol and IPQ-R responses, reported as relative risk ratios (RRR) with $95 \%$ confidence intervals $(95 \% \mathrm{CI})$. After initial unadjusted analysis, RRR were adjusted for the sample characteristics of age, gender and deprivation status, BMI, alcohol consumption and comorbidities. The choice of these relating to their association with illness perceptions $[8,12]$ and gout $[13,14]$. A subsequent adjustment was then made for gout characteristics, an important confounder likely to influence patients' perceptions of their illness [8]. Gout characteristic included; the number of gout attacks in previous 12 months, history of oligo/polyarticular attacks, age at gout diagnosis and whether currently experiencing a gout attack.

Model diagnostics such as Nagelkerke's Pseudo $\mathrm{R}^{2}$ and Pearson goodness-of- fit values are reported for the latter models in order to show that the models are a reasonable approximation of the data. Nagelkerke's Rsquared reflects the change in terms of log-likelihood from the intercept-only model to the current model. It does not convey the same information as the R-square for linear regression. All analyses were undertaken using SPSS v.21.

\section{Results}

\section{Sample characteristics}

Detailed characteristics of this sample have been reported elsewhere [15], but in brief, the 1184 responders to the baseline questionnaire (65.6\%) had a mean age of 65.6 years $(\mathrm{SD}=12.5)$, the majority were male $(83.6 \%)$ and classified as overweight or obese (68.7\%). Nearly a quarter of participants drank alcohol on a daily basis (23.4 \%) and the most prevalent comorbidities were hypertension (61.7 \%), and hyperlipidaemia (42.9 \%) (Table 1).

The average age at which gout had been diagnosed was 53.4 years (SD 15.9) and 725 (64.5\%) patients had experienced at least one gout attack in the previous 12 months. One hundred thirty-two patients (11.1\%) were experiencing an attack of gout at the time of completing the baseline questionnaire and $436(36.8 \%)$ had a history of oligo/polyarticular attacks. Just over half of baseline responders $(56.3 \%)$ were using allopurinol (Table 2). When stratified by allopurinol use, there was no difference in age, alcohol consumption, social deprivation
Table 1 General characteristics

\begin{tabular}{lccc}
\hline & & \multicolumn{2}{c}{ Using allopurinol } \\
\cline { 3 - 4 } Factor & Frequency (\%) & No (\%) & Yes (\%) \\
\hline Age Mean (SD) & $65.6(12.5)$ & $65.1(13.4)$ & $65.9(11.6)$ \\
Gender (Male \%) & $990(83.6)$ & $390(79.6)$ & $544(86.4)$ \\
Alcohol Consumption & & & \\
$\quad$ Never & $113(9.7)$ & $50(10.4)$ & $56(9.0)$ \\
Occasionally & $155(13.3)$ & $63(13.1)$ & $86(13.8)$ \\
1-3 times per month & $109(9.3)$ & $47(9.8)$ & $57(9.0)$ \\
1-2 times per week & $254(21.8)$ & $110(22.9)$ & $129(20.6)$ \\
3-4 times per week & $263(22.5)$ & $102(21.3)$ & $148(23.7)$ \\
Daily & $273(23.4)$ & $108(22.5)$ & $149(23.8)$
\end{tabular}

Body Mass Index

$\begin{array}{llll}\text { Normal }\left(<25 \mathrm{~kg} / \mathrm{m}^{2}\right) & 371(31.3) & 168(34.3) & 178(28.2) \\ \text { Overweight/obese } & 813(68.7) & 322(65.7) & 452(71.8)\end{array}$
$\left(\geq 25 \mathrm{~kg} / \mathrm{m}^{2}\right)$

Neighbourhood Deprivation Status

$\begin{array}{lccc}20 \text { \% least deprived } & 410(34.6) & 174(35.5) & 214(34.0) \\ \text { Mid-deprived } & 405(34.2) & 160(32.7) & 228(36.2) \\ 20 \% \text { most deprived } & 369(31.2) & 156(31.8) & 188(29.8) \\ \text { Depression (PHQ-9) } & 131(11.1) & 46(11.0) & 76(13.4) \\ \text { Anxiety (GAD-7) } & 109(10.0) & 42(9.3) & 59(10.0) \\ \text { yype II Diabetes Mellitus } & 205(17.3) & 60(12.2) & 138(21.9) \\ \text { Stroke } & 37(3.1) & 12(2.5) & 24(3.8) \\ \text { Hypertension } & 731(61.7) & 274(55.9) & 414(65.7) \\ \text { Transient Ischaemic Attack } & 62(5.2) & 24(4.9) & 34(5.4) \\ \text { Hyperlipidaemia } & 508(42.9) & 190(38.8) & 291(46.2) \\ \text { Kidney Failure } & 56(4.7) & 18(3.7) & 31(4.9) \\ \text { Myocardial Infarction } & 119(10.1) & 44(9.0) & 67(10.6) \\ \text { Kidney Stones } & 81(6.8) & 23(4.7) & 51(8.1) \\ \text { Angina } & 147(12.4) & 49(10.0) & 88(14.0)\end{array}$

SD standard deviation, $P H Q-9$ personalised health questionnaire-9, GAD-7 generalised anxiety disorder 7 questionnaire

or the prevalence of anxiety, depression, stroke, TIA, kidney stones or MI between users and non-users. However, allopurinol users were more likely than non-users to be overweight $\left(\chi^{2}=4.70, \mathrm{df}=1120 p=.030\right)$ and to have diabetes $\left(\chi^{2}(1, N=1120)=17.67, p=<0.001\right)$, hypertension $\left(\chi^{2}=11.16, \mathrm{df}=1120, p=.010\right)$, hyperlipidaemia $\left(\chi^{2}=6.19\right.$, $\mathrm{df}=1120, p=.013)$, kidney stones $\left(\chi^{2}=5.17, \mathrm{df}=1120\right.$, $p=.023)$ and angina $\left(\chi^{2}=4.04, \mathrm{df}=1120, p=.044\right)$.

With regard to illness perceptions, approximately half of all participants agreed that there is either a lot they can do to control their gout $(51.2 \%)$ or that what they do will affect their gout (44.8\%). Threequarters of gout patients perceived treatments to be effective $(76.4 \%)$ and agreed that gout is a serious condition (76.4\%). 
Table 2 Gout characteristics

\begin{tabular}{lccc}
\hline & & \multicolumn{2}{c}{ Using allopurinol } \\
\cline { 3 - 4 } Characteristics & Frequency (\%) & No (\%) & Yes (\%) \\
\hline $\begin{array}{l}\text { Age at Gout Diagnosis } \\
\text { (Mean, SD) }\end{array}$ & $53.4(15.9)$ & $57.3(15.5)$ & $50.6(15.5)$ \\
Number of attacks in previous & & & \\
0 & $398(35.4)$ & $96(19.8)$ & $298(48.2)$ \\
$1-3$ & $521(46.4)$ & $253(52.2)$ & $163(26.4)$ \\
$\geq 4$ & $204(18.2)$ & $136(28.0)$ & $157(25.4)$ \\
Gout Attack at Present & $132(11.1)$ & $54(11.1)$ & $69(11.0)$ \\
History of oligo/Polyarticular & $436(36.8)$ & $141(29.0)$ & $283(45.3)$ \\
gout & & & \\
Currently Taking Allopurinol & & & - \\
$\quad$ Yes & $630(56.3)$ & - & - \\
$\quad$ No & $490(43.7)$ & - & - \\
\hline
\end{tabular}

Participants using allopurinol were diagnosed with gout at a younger mean age (50.6 years, SD 15.5) compared to non-users (57.3 years, SD 15.5), $t(1076)=-7.11, p<0.001$ (Table 2). Allopurinol users were significantly less likely to have experienced one or more attacks of gout in the preceding 12 months (51.8\% versus $80.2 \%)\left(\chi^{2}=95.63\right.$, $\mathrm{df}=1103, p<0.001$ ), but more likely to have a history of oligo/polyarticular attacks (45.3\% versus $29.0 \%)$ than non-users $\left(\chi^{2}=30.93, \mathrm{df}=1103, p<0.001\right)$.

\section{Associations between illness perceptions and use of allopurinol}

Unadjusted analysis showed that gout patients were more likely to agree (1.64 (1.14 to 2.34)) than disagree, with the statement that there is ' $a$ lot I can do to control my gout' when using allopurinol. This association was retained after initial adjustments (1.66 (1.12 to 2.45), but was attenuated after adjustments for gout characteristics (1.39 (0.89 to 2.17 ) (Table 3).

Gout patients were twice as likely to agree than disagree (unadjusted 2.20 (1.36 to 3.54), adjusted 2.24 (1.32 to 3.81$)$ ) that 'treatments are effective' if they were using allopurinol. However, this association was also attenuated when gout characteristics were adjusted for $(1.78$ (0.96 to 3.29). Both unadjusted and adjusted analysis showed no significant difference between the patient perception of 'What I do will affect my gout' or that "Gout is serious" and their current use of allopurinol.

The Pearson goodness of fit $p$-values provided no real cause for concern, except the borderline result for the

Table 3 Associations between Illness perceptions and allopurinol use

\begin{tabular}{|c|c|c|c|c|c|}
\hline & \multicolumn{2}{|c|}{ Using allopurinol } & \multirow[b]{2}{*}{ Unadjusted RRR (95\% Cl) } & \multicolumn{2}{|c|}{ Adjusted relative risk ratio (RRR) $(95 \%$ Cl) } \\
\hline & Yes (\%) & No (\%) & & $\begin{array}{l}\text { Age, gender, deprivation, BMI, } \\
\text { alcohol \& co-morbidities }\end{array}$ & $\begin{array}{c}\text { Age, gender, deprivation, BMI, } \\
\text { alcohol, co-morbidities \& gout } \\
\text { characteristics }\end{array}$ \\
\hline \multicolumn{6}{|l|}{ A lot I can do to control my gout } \\
\hline Disagree $(N=154)$ & $78(12.7)$ & $76(16.2)$ & REF & REF & REF \\
\hline Uncertain $(N=375)$ & $190(30.8)$ & $185(39.5)$ & $1.00(0.69$ to 1.46$)$ & $1.01(0.67$ to 1.51$)$ & $1.10(0.70$ to 1.73$)$ \\
\hline Agree $(N=555)$ & $348(56.5)$ & $207(44.3)$ & 1.64 (1.14 to 2.34$)$ & 1.66 (1.12 to 2.45$)$ & 1.39 (0.89 to 2.17$)$ \\
\hline Nagelkerke $r^{2}$, Pearson GOF & & & & $0.094, p=0.053$ & $0.18, p=0.43$ \\
\hline \multicolumn{6}{|l|}{ What I do will affect my gout } \\
\hline Disagree $(N=104)$ & $64(10.5)$ & $40(8.5)$ & REF & REF & REF \\
\hline Uncertain $(N=487)$ & $264(43.3)$ & $233(49.4)$ & 0.74 (0.48 to 1.14$)$ & $0.66(0.41$ to 1.04$)$ & 0.74 (0.44 to 1.23$)$ \\
\hline Agree $(N=480)$ & $281(46.2)$ & $199(42.1)$ & 0.88 (0.57 to 1.36$)$ & $0.82(0.51$ to 1.31$)$ & 0.69 (0.41 to 1.16$)$ \\
\hline Nagelkerke $r^{2}$, Pearson GOF & & & & $0.07, p=0.65$ & $0.12, p=0.91$ \\
\hline \multicolumn{6}{|l|}{ Treatments are effective } \\
\hline Disagree $(N=75)$ & $32(5.2)$ & $43(9.3)$ & REF & REF & REF \\
\hline Uncertain $(N=180)$ & $73(11.8)$ & $107(23.1)$ & 0.92 (0.53 to 1.58$)$ & 0.98 (0.54 to 1.79$)$ & 1.06 (0.54 to 2.06$)$ \\
\hline Agree $(N=827)$ & $513(83.0)$ & 314 (67.6) & 2.20 (1.36 to 3.54$)$ & $2.24(1.32$ to 3.81$)$ & $1.78(0.96$ to 3.29$)$ \\
\hline Nagelkerke $r^{2}$, Pearson GOF & & & & $0.12, p=0.11$ & $0.29, p=0.54$ \\
\hline \multicolumn{6}{|l|}{ Gout is serious } \\
\hline Disagree $(N=63)$ & $33(5.3)$ & $30(6.4)$ & REF & REF & REF \\
\hline Uncertain $(N=195)$ & $98(15.8)$ & 97 (20.6) & 0.92 (0.52 to 1.62$)$ & 0.79 (0.42 to 1.50$)$ & 0.91 (0.43 to 1.89$)$ \\
\hline Agree $(N=834)$ & $490(78.9)$ & $344(73.0)$ & 1.29 (0.78 to 2.16$)$ & 1.15 (0.65 to 2.05$)$ & 1.15 (0.59 to 2.26$)$ \\
\hline Nagelkerke $r^{2}$, Pearson GOF & & & & $0.09, p=0.39$ & $0.12, p=0.96$ \\
\hline
\end{tabular}


first adjusted model for ' $a$ lot I can do to control my gout'. Nagelkerke's R-squared are quite low suggesting other variables might need to be recorded.

\section{Discussion}

Our study examined the illness perceptions of gout patients from UK primary care and the associations between these perceptions and the current use of allopurinol. The majority of gout patients perceive that gout is a serious condition and that treatments are effective, but only half of patients believe that their actions have any influence over their own condition. Gout patients who feel more in control of their own condition and perceive treatments to be effective are more likely to be current users of allopurinol than non-users. Despite these associations being attenuated by gout characteristics, such links between illness perceptions and use of allopurinol remain worthy of reporting as this is likely an overadjustment, due to the close associations between illness perceptions, use of allopurinol and severity of gout.

Our overall findings that patients perceived gout to be a chronic condition that can be managed with treatment, but for which personal actions have little influence are in-line with those of Dalbeth et al. [8]. Furthermore, Dalbeth et al. also reported issues between the perception of control and ULT, as patients who had concerns over ULT had less ability to control their disease and recorded higher symptomatic scores in the B-IPQ. This may be reflected in our findings that those who perceived less control of their gout were less likely to be using allopurinol.

Two key factors which impair the effectiveness of allopurinol are poor adherence and sub-optimal dosing to sufficiently lower urate levels [1]. Informing and empowering patients in the control of their own disease may improve subsequent managements, such as use of allopurinol [16]. It is clear that patients need to be better informed of the long-term benefits of allopurinol, such as preventing irreversible joint damage and complications associated with comorbidities [17].

The strengths of this study are that this uses a large sample of gout patients from UK primary care, adding to the limited data of illness perceptions in gout patients. This also builds on research examining the current role of allopurinol in UK primary care.

Limitations of this study include the use of only four IPQ- $R$ questions from the responder questionnaire; however, these questions were selected on the basis that they most appropriately represented patient perceptions of gout and ULT, based on our previous qualitative research with gout patients [18]. One of these IPQ-R questions refers to treatment, but does not mention allopurinol or ULT specifically. In determining the perceptions of treatments in allopurinol users, we have assumed that patients taking allopurinol answered the treatment question with this in mind, rather than other treatments. As we specifically asked patients whether they were "currently taking a tablet called allopurinol", then it is likely that this is their principal treatment (as guidelines recommend) and that they were at least adhering at the point of the baseline questionnaire. The vast majority of ULT users in primary care in the UK are prescribed allopurinol, with other ULTs (febuxostat and uricosurics) comprising less than $2 \%$ of prescriptions for ULT in 2015 [19]. Whilst we believe that any perceptions about ULT will relate to allopurinol, we did not quantify use of, or ask about perceptions of other drugs such as colchicine or NSAIDs. We are also unable to determine whether the reasons behind non-allopurinol use are related to patient (e.g. poor adherence) or practitioner reasons (e.g. lack of provision by the general practitioner (GP)). Finally, our findings may be an effect of confounding by indication. It is likely that those prescribed allopurinol have the most severe gout, supported by our finding that allopurinol users more frequently reported experiencing oligo/polyarticular attacks in the past than non-users. Therefore, allopurinol may be acting as a proxy for increased disease severity.

Improved gout characteristics, be this due to allopurinol use or not, are the desired goals. If patients perceive that they are able to control and treat their disease, this may encourage improved adherence and continued optimal management. In light of these findings and that only half $(53.2 \%)$ of this gout sample were using allopurinol, the key clinical implication of our findings is a need to ensure that clinicians are appropriately trained to discuss the use of ULT with patients, and that patients are given highquality information to aid their understanding of the benefits of ULT and importance of adherence to treatment.

\section{Conclusions}

In conclusion, gout patients in UK primary care understand the seriousness of their condition and that treatments are effective, but perceive little personal control of their condition. Gout patients who viewed gout treatments as effective and did perceive a sense of control were more likely to be currently using allopurinol. Therefore, better information is needed for the patient, from GPs and rheumatologist, to encourage a sense of control over one's condition with the aim improving health outcomes.

\footnotetext{
Abbreviations

B-IPQ: Brief illness perception questionnaire; BMI: Body mass index; CLAHRC: Collaborations for leadership in applied health research and care; GAD: Generalised anxiety disorder; GP: General practitioner; HAQ II: Health assessment questionnaire; IMD: Indices of multiple deprivation; IPQR: Revised illness perception questionnaire; MSU: Monosodium urate; MTPJ: Metatarsophalangeal joint; NIHR: National Institute for Health Research; PHQ: Patient health questionnaire; R-IPQ: Revised illness perception questionnaire; RRR: Relative risk ratios; SD: Standard deviation; sUA: Serum urate; ULT: Urate-lowering therapies
} 


\section{Acknowledgements}

We are grateful for the participation of general practice teams and their patients. Acknowledgements are given to the Keele survey, network, administration and management teams who supported the study.

\section{Funding}

CPW was funded through an INSPIRE Summer Studentship, coordinated by the Academy of Medical Sciences and funded by the Wellcome Trust. JAP is funded by a Launching Fellowship from the NIHR School for Primary Care Research. PC is funded by a Doctoral Training Fellowship from the NIHR School for Primary Care Research. CDM is funded by the National Institute for Health Research (NIHR) Collaborations for Leadership in Applied Health Research and Care (CLAHRC) West Midlands, the NIHR School for Primary Care Research and a NIHR Research Professorship in General Practice (NIHR-RP-2014-04-026).

The study sponsors had no role in study design; in the collection, analysis, and interpretation of data; in the writing of the report; and in the decision to submit the paper for publication. The views and opinions expressed therein are those of the authors and do not necessarily reflect those of the NIHR (UK). This paper presents independent research which is part-funded by the CLAHRC West Midlands. The views expressed are those of the author(s) and not necessarily those of the NHS, the NIHR or the Department of Health.

\section{Availability of data and materials}

Data is available through formal data request processes (www.keele.ac.uk/ pchs/publications/datasharingresources/.

\section{Authors' contributions}

Guarantor of overall study integrity: JP, CDM \& ER. Study concept \& design: ER, CDM, PC. Data collection and interpretation: JAP, PC, ER \& CDM. Statistical analysis: CPW, JAP, JB. Manuscript preparation: CPW, JAP, JB, CDM \& ER. Final approval of manuscript: CPW, JAP, PC, JB, CDM, ER.

\section{Competing interests}

The authors declare that they have no competing interests.

\section{Consent for publication}

Not applicable.

\section{Ethics approval and consent to participate}

Full ethical approval was granted to this study by the North West - Liverpool East Research Ethics Committee (Ref no: 12/NW/0297) and all subjects provided informed consent to participate.

Received: 10 August 2016 Accepted: 13 September 2016 Published online: 17 September 2016

\section{References}

1. Kuo C, Grainge MJ, Mallen C, Zhang W, Doherty M. Rising burden of gout in the UK but continuing suboptimal management: a nationwide population study. Ann Rheum Dis. 2015;74:661-7.

2. Roddy E, Mallen CD, Doherty M. Gout BMJ. 2013;347:f5648.

3. Jordan KM, Cameron JS, Snaith M, Zhang W, Doherty M, Seckl J, Hingorani A, Jaques R, Nuki G, British Society for Rheumatology and British Health Professionals in Rheumatology Standards, Guidelines and Audit Working Group (SGAWG). British Society for Rheumatology and British Health Professionals in Rheumatology guideline for the management of gout. Rheumatology (Oxford). 2007:46(8):1372-4

4. Petrie KJ, Weinman J. Why illness perceptions matter. Clin Med. 2006;6(6):536-9

5. Moss-Morris R, Weinman J, Petrie K, Horne R, Cameron L, Buick D. The revised illness perception questionnaire (IPQ-R). Psychol Health. 2002:17(1):1-16

6. Leventhal H, Benyami Y, Brownlee S, Diefenbach M, Leventhal EA, PatrickMiller L. Illness Representations: Theoretical Foundations. In: Anonymous Perceptions of health and illness. London: Harwood Publishers; 1997. p. 19

7. Lau RR, Bernard TM, Hartman KA. Further explorations of common-sense representations of common illnesses. Health Psychol. 1989;8(2):195-219.

8. Dalbeth N, Petrie KJ, House M, Chong J, Leung W, Chegudi R, Horne A, Gamble G, McQueen FM, Taylor WJ. Illness perceptions in patients with gout and the relationship with progression of musculoskeletal disability. Arthritis Care Res. 2011:63(11):1605-12.

9. De Gucht V. Illness perceptions mediate the relationship between bowel symptom severity and health-related quality of life in IBS patients. Qual Life Res. 2015;24(8):1845-56.

10. Kessels RPC. Patients' memory for medical information. J R Soc Med. 2003;96(5):219-22

11. Chandratre P, Mallen C, Richardson J, Rome K, Bailey J, Gill R, Hider S, Mason J, Mayson Z, Muller S, Purcell C, Titley J, Wathall S, Zwierska I, Roddy E. Prospective observational cohort study of Health Related Quality of Life (HRQOL), chronic foot problems and their determinants in gout: a research protocol. BMC Musculoskelet Disord. 2012;13(1):219.

12. Saarti S, Hajj A, Karam L, Jabbour H, Sarkis A, El Osta N, Rabbaa Khabbaz L. Association between adherence, treatment satisfaction and illness perception in hypertensive patients. J Hum Hypertens. 2016:30(5):341-5.

13. Prior JA, Mallen CD, Chandratre P, Muller S, Richardson J, Roddy E. Gout characteristics associate with depression, but not anxiety, in primary care: Baseline findings from a prospective cohort study. Joint Bone Spine. 2016. in Press.

14. Kuo C, Grainge MJ, Mallen C, Zhang W, Doherty M. Comorbidities in patients with gout prior to and following diagnosis: case-control study. Ann Rheum Dis. 2016:75(1):210-7.

15. Roddy E, Muller S, Rome K, Chandratre P, Hider SL, Richardson J, Blagojevic-Bucknall M, Mallen CD. Foot problems in people with gout in primary care: baseline findings from a prospective cohort study. J Foot Ankle Res. 2015; In press.

16. Harrold LR, Mazor KM, Velten S, Ockene IS, Yood RA. Patients and providers view gout differently: A qualitative study. Chronic IIIn. 2010;6(4):263-71.

17. Doherty M, Jansen TL, Nuki G, Pascual E, Perez-Ruiz F, Punzi L, So AK, Bardin T. Gout: why is this curable disease so seldom cured? Ann Rheum Dis. 2012:71(11):1765-70.

18. Liddle J, Roddy E, Mallen CD, Hider SL, Prinjha S, Ziebland S, Richardson JC. Mapping patients' experiences from initial symptoms to gout diagnosis: a qualitative exploration. BMJ Open. 2015;5(9):e008323.

19. Prescribing \& Medicines Team Health and Social Care Information Centre. Prescription Cost Analysis England 2015. 2016. http://digital.nhs.uk/article/ 2021/WebsiteSearch?productid $=20437 \& q=$ drugs\&topics=13210\&infotype= 13367\&sort=Relevance\&size=10\&page=1\#top. Accessed 1 June 2016.

\section{Submit your next manuscript to BioMed Central and we will help you at every step:}

- We accept pre-submission inquiries

- Our selector tool helps you to find the most relevant journal

- We provide round the clock customer support

- Convenient online submission

- Thorough peer review

- Inclusion in PubMed and all major indexing services

- Maximum visibility for your research

Submit your manuscript at www.biomedcentral.com/submit
Biomed Central 